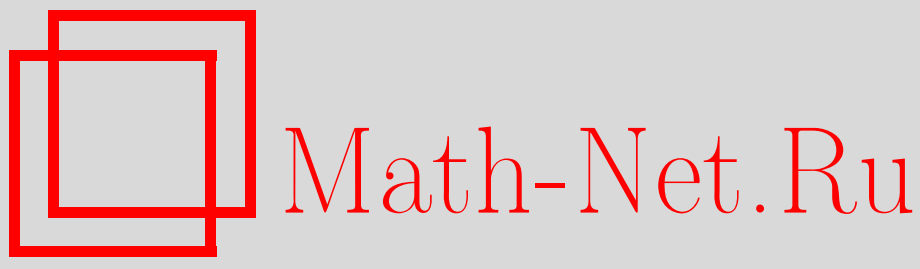

В. В. Сенатов, Несколько асимптотических разложений в ЦПТ в многомерном случае, Теория вероятн. и ее примен., 2008, том 53, выпуск 2, 293-306

DOI: https://doi.org/10.4213/tvp2410

Использование Общероссийского математического портала Math-Net.Ru подразумевает, что вы прочитали и согласны с пользовательским соглашением

http://www . mathnet.ru/rus/agreement

Параметры загрузки:

IP : 54.198 .187 .58

26 апреля 2023 г., $17: 26: 45$

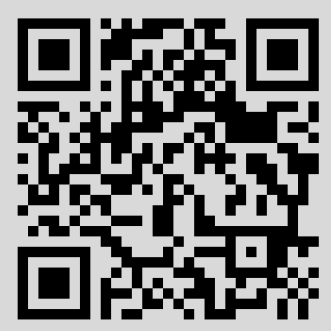




\title{
НЕСКОЛЬКО АСИМПТОТИЧЕСКИХ РАЗЛОЖЕНИЙ В ЦПТ В МНОГОМЕРНОМ СЛУЧАЕ ${ }^{1)}$
}

\begin{abstract}
Получены асимптотические разложения в центральной предельной теореме в многомерном случае с явными оценками точности аппроксимации, которую они гарантируют. Рассматриваются нормированные суммы независимых одинаково распределенных случайных величин с конечными моментами четвертого и пятого порядка. При построении разложений используются многомерные аналоги многочленов ЧебышёваЭрмита.
\end{abstract}

Ключевые слова и фразы: центральная предельная теорема, многомерные распределения, асимптотические разложения, многомерные аналоги многочленов Чебышёва-Эрмита.

Цель данной работы - продемонстрировать несколько асимптотических разложений в ЦПТ в многомерных пространствах и дать явные оценки точности аппроксимации, которую гарантируют эти разложения.

Пусть $X_{1}, X_{2}, \ldots$ - независимые одинаково распределенные случайные величины с нулевым средним и единичным ковариационным оператором, принимаюшие значения в действительном евклидовом $d$-мерном пространстве $E^{d}$. Пусть $P$ - общее распределение этих случайных величин, $P_{n}-$ распределение нормированной суммы $\left(X_{1}+\cdots+X_{n}\right) / \sqrt{n}$, $p_{n}$ - плотность $P_{n}$ (если она существует), $\Phi$ - нормальный закон с нулевым средним и единичным ковариационным оператором, $\varphi(x)=$ $(2 \pi)^{-d / 2} e^{-|x|^{2} / 2}$ - плотность $\Phi$. Здесь и далее $|x|$ означает как абсолютную величину числа $x$, так и норму вектора $x \in E^{d}$.

Предположим, что характеристическая функция

$$
f(t)=\int e^{i(t, x)} P(d x), \quad t \in E^{d},
$$

распределения $P$ такова, что для некоторого числа $\nu>0$

$$
\int|f(t)|^{\nu} d t<\infty
$$

* Московский государственный университет им. М.В. Ломоносова, механикоматематический факультет, кафедра теории вероятностей, Ленинские горы, 119992 Москва, Россия; e-mail: semenova@greatbook.ru

1) Работа выполнена при поддержке РФФИ (гранты 05-01-00583, 07-01-00362). 
Здесь и далее $(t, x)$ обозначает скалярное произведение векторов $t, x \in E^{d}$; если область интегрирования не указывается, то она совпадает со всем пространством $E^{d}$. Выполнение условия (1) гарантирует существование непрерывной и ограниченной плотности $p_{n}$ для $n \geqslant \nu$, ее можно вычислять по формуле обращения

$$
p_{n}(x)=(2 \pi)^{-d} \int e^{-i(t, x)} f^{n}\left(\frac{t}{\sqrt{n}}\right) d t .
$$

Нетрудно проверить, что выполнение условия (1) гарантирует выполнение (строгого) неравенства

$$
\alpha(T)=\max \{|f(t)|:|t| \geqslant T\}<1
$$

для любого $T>0$.

Асимптотические разложения, приведенные ниже, основаны на многомерных аналогах

$$
H_{l}^{(2 j)}(x)\left(h_{1}, \ldots, h_{l}\right), \quad j=0,1,2, \ldots, \quad l=0,1,2, \ldots,
$$

многочленов Чебышёва-Эрмита. Эти аналоги предложены в работе [1], они являются полилинейными функционалами, действие $H_{l}^{(2 j)}(x)$ на $h_{1}, \ldots, h_{l} \in E^{d}$ можно получать по формуле обращения

$$
H_{l}^{(2 j)}(x)\left(h_{1}, \ldots, h_{l}\right) \varphi(x)=\frac{i^{2 j+l}}{(2 \pi)^{d}} \int e^{-i(t, x)}|t|^{2 j} e^{-|t|^{2} / 2}\left(t, h_{1}\right) \cdots\left(t, h_{l}\right) d t .
$$

Для каждого $e \in E_{d},|e|=1$, обозначим

$$
\begin{aligned}
\alpha_{k}(e) & =\int(x, e)^{k} P(d x) \quad\left(=\mathbf{E}\left(X_{1}, e\right)^{k}\right), \\
\beta_{k}(e) & =\int\left|(x, e)^{k}\right| P(d x) \quad\left(=\mathbf{E}\left|\left(X_{1}, e\right)\right|^{k}\right), \\
\left|\alpha_{k}\right| & =\sup _{|e|=1}\left|\alpha_{k}(e)\right|, \quad\left|\beta_{k}\right|=\sup _{|e|=1}\left|\beta_{k}(e)\right|, \quad k=0,1,2, \ldots, \\
\left|\theta_{4}\right| & =\sup _{|e|=1}\left|\alpha_{4}(e)-3\right| .
\end{aligned}
$$

Всюду, где эти величины используются, предполагается, что они конечны. Отметим, что $\alpha_{k}(e)$ и $\beta_{k}(e)$ представляют собой $k$-й и $k$-й абсолютный моменты проекции распределения $P$ на направление $e$; записи $\left|\alpha_{k}\right|$ и $\left|\theta_{4}\right|$ являются едиными, мы нигде не будем использовать $\alpha_{k}$ и $\theta_{4}$ без знака модуля. Отметим, что $\left|\alpha_{k}\right|=\beta_{k}$ для четных $k$.

Зафиксируем некоторое число $0<\delta<1$. С помощью известного приема симметризации нетрудно проверить, что если $\beta_{4}<\infty$, то для всех $|t| \leqslant T$ справедливо неравенство $|f(t)| \leqslant \mu(t)$, где

$$
\mu(t)=e^{-\left(1-\delta^{2}\right)|t|^{2} / 2}, \quad T=\min \left(1, \delta\left(\frac{12}{\beta_{4}+3}\right)^{1 / 2}\right) .
$$


Положим

$$
\begin{aligned}
B_{k} & =(2 \pi)^{-d} \int|t|^{k} e^{-|t|^{2} / 2} d t, \\
B_{k, n} & =(2 \pi)^{-d} \int_{|t| \leqslant T \sqrt{n}}|t|^{k} \mu^{n}\left(\frac{t}{\sqrt{n}}\right) d t, \\
B_{k, n-1} & =(2 \pi)^{-d} \int_{|t| \leqslant T \sqrt{n}}|t|^{k} \mu^{n-1}\left(\frac{t}{\sqrt{n}}\right) d t .
\end{aligned}
$$

Для чисел $B_{k}, B_{k, n}$ и $B_{k, n-1}$ нетрудно получить явные выражения и оценки, но они нам не понадобятся. Очевидно, что при малых $\delta$ и больших $n$ числа $B_{k, n}$ и $B_{k, n-1}$ близки к $B_{k}$.

Теорема 1. Пусть распределение $P$ таково, что $\beta_{4}<\infty$ и для некоторого $\nu>0$ выполнено условие (1). Тогда для любого $n \geqslant$ $\max (\nu, 2)$ и всех $x \in E^{d}$ справедливо равенство

$$
p_{n}(x)=\varphi(x)+\frac{\varphi(x)}{6 \sqrt{n}} \int\left((x, u)^{3}-3(x, u)(u, u)\right) P(d u)+R,
$$

2дe

$$
\begin{aligned}
|R| \leqslant & \frac{\beta_{4}+3}{4 !} \frac{B_{4, n-1}}{n}+\frac{1}{2}\left(\frac{\left|\alpha_{3}\right|}{3 !}\right)^{2} \frac{B_{6}}{n} \\
& +\frac{\left|\alpha_{3}\right|}{12} \frac{B_{5, n-1}}{n^{3 / 2}}+\frac{1}{2}\left(\frac{\left|\alpha_{3}\right|}{3 !}\right)^{2} \frac{B_{6, n}}{n^{2}} \\
& +\frac{n^{d / 2}}{(2 \pi)^{d}} \alpha^{n-\nu}(T) \int_{|t| \geqslant T}|f(t)|^{\nu} d t+\frac{1}{(2 \pi)^{d}} \int_{|t| \geqslant T \sqrt{n}} e^{-|t|^{2} / 2} d t .
\end{aligned}
$$

Отметим, что подынтегральное выражение в правой части (3) совпадает с многочленом $H_{3}^{(0)}(x)(u, u, u)$; в одномерном случае этот интеграл распадается в произведение многочлена Чебышёва-Эрмита 3-го порядка и момента Чебышёва-Эрмита 3-го порядка распределения $P$ (см. [2]).

В оценке (4) остаточной части разложения (3) несколько слагаемых убывают при росте $n$ как $1 / n$. Некоторые из этих слагаемых можно «перевести» (с соответствующими изменениями) в главную часть разложения плотности $p_{n}$, улучшив при этом оценку остаточной части (во всяком случае при больших $n$ ).

В следующих утверждениях нам понадобится величина

где

$$
H_{6}(x ; P)=\iint H_{6}^{(0)}(x)(u, u, u, v, v, v) P(d u) P(d v),
$$

$$
\begin{aligned}
& H_{6}^{(0)}(x)(u, u, u, v, v, v)=(x, u)^{3}(x, v)^{3}-3(u, u)(u, x)(v, x)^{3} \\
& \quad-9(u, x)^{2}(u, v)(x, v)^{2}-3(u, x)^{3}(x, v)(v, v)+9(u, u)(u, v)(x, v)^{2} \\
& \quad+9(u, x)^{2}(u, v)(v, v)+9(u, x)(u, u)(v, v)(x, v)+18(u, x)(u, v)^{2}(x, v) \\
& \quad-6(u, v)^{3}-9(u, u)(u, v)(v, v) .
\end{aligned}
$$


Отметим, что в одномерном случае $H_{6}(x ; P)$ распадается в произведение многочлена Чебышёва-Эрмита 6-го порядка и квадрата момента Чебышёва-Эрмита 3-го порядка распределения $P$.

Теорема 2. При выполнении условий теоремы 1 для всех $x \in E^{d}$ справедливо равенство

$$
p_{n}(x)=\varphi(x)+\frac{\varphi(x)}{6 \sqrt{n}} \int H_{3}^{(0)}(x)(u, u, u) P(d u)+\frac{\varphi(x)}{72 n} H_{6}(x ; P)+R,
$$

2de

$$
\begin{aligned}
|R| \leqslant & \frac{\beta_{4}+3}{4 !} \frac{B_{4, n-1}}{n}+\frac{1}{3 !}\left(\frac{\left|\alpha_{3}\right|}{3 !}\right)^{3} \frac{B_{9}}{n^{3 / 2}}+\frac{\left|\alpha_{3}\right|}{12} \frac{B_{5, n-1}}{n^{3 / 2}}+\frac{1}{2}\left(\frac{\left|\alpha_{3}\right|}{3 !}\right)^{2} \frac{B_{6, n}}{n^{2}} \\
& +\frac{n^{d / 2}}{(2 \pi)^{d}} \alpha^{n-\nu}(T) \int_{|t| \geqslant T}|f(t)|^{\nu} d t+\frac{1}{(2 \pi)^{d}} \int_{|t| \geqslant T \sqrt{n}} e^{-t^{2} / 2} d t
\end{aligned}
$$

и если величина $n \geqslant \max (\nu, 3)$ такова, что

mo

$$
\rho=\frac{1}{2}+\frac{d-1}{n}+\frac{d(d+2)}{24 n}<1,
$$

$$
\begin{aligned}
p_{n}(x)= & \varphi(x)+\frac{\varphi(x)}{6 \sqrt{n}} \int H_{3}^{(0)}(x)(u, u, u) P(d u) \\
& -\frac{\varphi(x)}{8 n}\left(|x|^{4}-2(d+2)|x|^{2}+d(d+2)\right)+\frac{\varphi(x)}{72 n} H_{6}(x ; P)+R,
\end{aligned}
$$

¿de

$$
\begin{aligned}
|R| \leqslant & \frac{\beta_{4}}{4 !} \frac{B_{4, n-1}}{n}+\frac{1}{3 !}\left(\frac{\left|\alpha_{3}\right|}{3 !}\right)^{3} \frac{B_{9}}{n^{3 / 2}}+\frac{\left|\alpha_{3}\right|}{12} \frac{B_{5, n-1}}{n^{3 / 2}}+\frac{\left|\alpha_{3}\right|}{48} \frac{B_{7}}{n^{3 / 2}}+\frac{B_{6, n-1}}{12 n^{2}} \\
& +\frac{1}{2}\left(\frac{\left|\alpha_{3}\right|}{3 !}\right)^{2} \frac{B_{6, n}}{n^{2}}+\frac{1}{128(1-\rho)} \frac{B_{8}}{n^{2}}+\frac{\left|\alpha_{3}\right|}{48} \frac{B_{7, n}}{n^{5 / 2}} \\
& +\frac{n^{d / 2}}{(2 \pi)^{d}} \alpha^{n-\nu}(T) \int_{|t| \geqslant T}|f(t)|^{\nu} d t \\
& +\frac{n^{d / 2}}{(2 \pi)^{d}} \beta^{n-1}(T) \int_{|t| \geqslant T} e^{-|t|^{2} / 2}\left|1-\frac{|t|^{4}}{8}\right| d t
\end{aligned}
$$

$u$

$$
\beta(T)=\max \left\{e^{-|t|^{2} / 2}\left|1-\frac{|t|^{4}}{8}\right|:|t| \geqslant T\right\}<1 .
$$

Отметим, что многочлен $|x|^{4}-2(d+2)|x|^{2}+d(d+2)=H_{0}^{(4)}(x)$ одномерном случае совпадает с многочленом Чебышёва-Эрмита четвертого порядка.

Теорема 3. Пусть распределение $P$ таково, что $\beta_{5}<\infty, \beta_{4}<9$ $u$ для некоторого $\nu>0$ выполнено условие (1). Тогда для всех $n \geqslant$ $\max (\nu, 3)$ makux, umo

$$
\rho=\frac{\left|\theta_{4}\right|}{6}+\frac{\left|\theta_{4}\right|(d-1)}{3 n}+\frac{\left|\theta_{4}\right| d(d+2)}{72 n}<1
$$


при всех $x \in E^{d}$ справедливо равенство

$$
\begin{aligned}
p_{n}(x)= & \varphi(x)+\frac{\varphi(x)}{6 \sqrt{n}} \int H_{3}^{(0)}(x)(u, u, u) P(d u) \\
+ & \frac{\varphi(x)}{24 n}\left\{\int\left((x, u)^{4}-6(x, u)^{2}(u, u)+3(u, u)^{2}\right) P(d u)\right. \\
& \left.\quad-3\left(|x|^{4}-2(d+2)|x|^{2}+d(d+2)\right)\right\} \\
+ & \frac{\varphi(x)}{72 n} H_{6}(x ; P)+R
\end{aligned}
$$

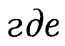

$$
\begin{aligned}
|R| \leqslant & \left(\frac{\beta_{5}}{5 !}+\frac{\left|\alpha_{3}\right|}{12}\right) \frac{B_{5, n-1}}{n^{3 / 2}}+\frac{\left|\alpha_{3}\right|}{3 !} \frac{\left|\theta_{4}\right|}{4 !} \frac{B_{7}}{n^{3 / 2}}+\frac{1}{3 !}\left(\frac{\left|\alpha_{3}\right|}{3 !}\right)^{3} \frac{B_{9}}{n^{3 / 2}} \\
& +\frac{\left|\alpha_{4}\right|+4}{48} \frac{B_{6, n-1}}{n^{2}}+\frac{1}{2}\left(\frac{\left|\alpha_{3}\right|}{3 !}\right)^{2} \frac{B_{6, n}}{n^{2}}+\frac{1}{2(1-\rho)}\left(\frac{\left|\theta_{4}\right|}{4 !}\right)^{2} \frac{B_{8}}{n^{2}} \\
& +\frac{\left|\alpha_{3}\right|}{3 !} \frac{\left|\theta_{4}\right|}{4 !} \frac{B_{7, n}}{n^{5 / 2}}+\frac{n^{d / 2}}{(2 \pi)^{d}} \alpha^{n-\nu}(T) \int_{\mid t \geqslant T}|f(t)|^{\nu} d t \\
& +\frac{n^{d / 2}}{(2 \pi)^{d}} \beta^{n-1}(T) \int_{|t| \geqslant T} e^{-|t|^{2} / 2}\left(1+\frac{\left|\theta_{4}\right|}{4 !}|t|^{4}\right) d t
\end{aligned}
$$

$u$

$$
\beta(T)=\max \left\{e^{-|t|^{2} / 2}\left(1+\frac{\left|\theta_{4}\right|}{4 !}|t|^{4}\right):|t| \geqslant T\right\}<1 .
$$

Отметим, что подынтегральное выражение во втором интеграле в правой части (9) совпадает с $H_{4}^{(0)}(x)(u, u, u, u)$. В одномерном случае величина в фигурных скобках в правой части (9) распадается в произведение многочлена Чебышёва-Эрмита четвертого порядка и момента Чебышёва-Эрмита четвертого порядка распределения $P$. Величина

$$
\frac{1}{4 !}\left\{\int H_{4}^{(0)}(x)(u, u, u, u) P(d u)-3 H_{0}^{(4)}(x)\right\},
$$

которая, с точностью до обозначений, присутствует в правой части (9), совпадает (см. [1]) с

$$
\begin{aligned}
& \int \frac{H H_{4}(x, u)}{4 !} P(d u) \\
&=\int\left(\frac{H_{4}^{(0)}(x)(u, u, u, u)}{4 !}-\frac{H_{2}^{(2)}(x)(u, u)}{2 ! 2^{1} \cdot 1 !}+\frac{H_{0}^{(4)}(x)}{0 ! 2^{2} \cdot 2 !}\right) P(d u),
\end{aligned}
$$

так как интегрирование $H_{2}^{(2)}(x)(u, u)=(x, x)(x, u)^{2}-(d+4)(x, u)^{2}-$ $(x, x)(u, u)+(d+2)(u, u)$ по мере $P(d u)$ с нулевым средним и единичным ковариационным оператором дает многочлен $H_{0}^{(4)}(x)$. Стоит отметить также, что

$$
\int(x, u)^{4} P(d u)-\mathbf{E}\left(x, X_{1}\right)^{4}=|x|^{4} \alpha_{4}\left(e_{x}\right), \quad \int(u, u)^{2} P(d u)=\mathbf{E}\left|X_{1}\right|^{4},
$$


a

$$
\int(x, u)^{2}(u, u) P(d u)=|x|^{2} \mathbf{E}\left(e_{x}, X_{1}\right)^{2}\left|X_{1}\right|^{2},
$$

где $e_{x}=x /|x|, x \in E^{d}$, и последнее математическое ожидание есть некий смешанный момент четвертого порядка случайной величины $X_{1}$, т.е. второй интеграл в правой части (9) связан с различными моментами четвертого порядка распределения $P$. Аналогичные слова можно сказать обо всех интегралах, участвующих в главных частях приведенных асимптотических разложений: все эти интегралы суть функции $|x|$ и различных моментных характеристик распределения $P$. Таким образом, как и в одномерном случае, мы вычисляем функцию $p_{n}(x)$ (разумеется, лишь с некоторой точностью) по нескольким, довольно грубым, моментным характеристикам распределения $P$.

Для доказательства сформулированных выше утверждений нам понадобится следуюшее утверждение.

Лемма 1. Для произведения $e^{|t|^{2} / 2} f(t)$, где $f$ - характеристическая функиия распределения $P$, при всех $t \in E^{d}$ в случае, когда конечна величина $\beta_{4}$, справедливы представления

$$
e^{|t|^{2} / 2} f(t)=1+\frac{i^{3} \alpha_{3}\left(e_{t}\right)|t|^{3}}{3 !}+\gamma e^{|t|^{2} / 2} \frac{\beta_{4}+3}{4 !}|t|^{4}+\gamma e^{|t|^{2} / 2} \frac{\left|\alpha_{3}\right|}{12}|t|^{5}
$$

$u$

$$
\begin{aligned}
e^{|t|^{2} / 2} f(t)= & 1+\frac{i^{3} \alpha_{3}\left(e_{t}\right)|t|^{3}}{3 !}-\frac{|t|^{4}}{8}+\gamma e^{|t|^{2} / 2} \frac{\beta_{4}}{4 !}|t|^{4} \\
& +\gamma e^{|t|^{2} / 2} \frac{\left|\alpha_{3}\right|}{12}|t|^{5}+\gamma e^{|t|^{2} / 2} \frac{|t|^{6}}{12}
\end{aligned}
$$

а в случае, когда $\beta_{5}<\infty$, для этого произведения справедливо представление

$$
\begin{aligned}
e^{|t|^{2} / 2} f(t)= & 1+\frac{i^{3} \alpha_{3}\left(e_{t}\right)|t|^{3}}{3 !}+\frac{i^{4}\left(\alpha_{4}\left(e_{t}\right)-3\right)|t|^{4}}{4 !} \\
& +\gamma e^{|t|^{2} / 2}\left(\frac{\beta_{5}}{5 !}+\frac{\left|\alpha_{3}\right|}{12}\right)|t|^{5}+\gamma e^{|t|^{2} / 2} \frac{\left|\alpha_{4}\right|+4}{48}|t|^{6} .
\end{aligned}
$$

Здесь и далее $e_{t}=t /|t|$, а $\gamma$ обозначают непрерывные комплекснозначные функиии такие, что $|\gamma| \leqslant 1$.

Д о к а з а т е л с с в о. Функцию $f(t)$ можно представить в виде

$$
\begin{aligned}
f(t) & =\int e^{i(t, x)} P(d x) \\
& =\int\left(1+i(t, x)+\frac{i^{2}}{2}(t, x)^{2}+\frac{i^{3}}{3 !}(t, x)^{3}+\frac{\gamma}{4 !}(t, x)^{4}\right) P(d x) \\
& =1-\frac{(t, t)}{2}+\frac{i^{3}}{3 !} \int(t, x)^{3} P(d x)+\frac{1}{4 !} \int \gamma(t, x)^{4} P(d x) \\
& =1-\frac{(t, t)}{2}+\frac{i^{3}|t|^{3} \alpha_{3}\left(e_{t}\right)}{3 !}+\frac{\gamma}{4 !}|t|^{4} \beta_{4} .
\end{aligned}
$$


Третье равенство в этой цепочке справедливо в силу того, что распределение $P$ имеет нулевое среднее и единичный ковариационный оператор. Во избежание недоразумений отметим, что функции $\gamma$ в левой и правой частях последнего равенства различны.

Рассмотрим произведение

$$
\begin{aligned}
e^{|t|^{2} / 2} f(t) & =e^{|t|^{2} / 2}\left(1-\frac{|t|^{2}}{2}+\frac{i^{3}|t|^{3} \alpha_{3}\left(e_{t}\right)}{3 !}+\frac{\gamma}{4 !}|t|^{4} \beta_{4}\right) \\
& =e^{|t|^{2} / 2}\left(1-\frac{|t|^{2}}{2}\right)+e^{|t|^{2} / 2} \frac{i^{3}|t|^{3} \alpha_{3}\left(e_{t}\right)}{3 !}+\frac{\gamma}{4 !} e^{|t|^{2} / 2}|t|^{4} \beta_{4} .
\end{aligned}
$$

Для любого $u>0$

$$
e^{-u}=1-e^{-\theta u} u,
$$

где $0<\theta<1$. Умножая это равенство на $e^{u}$ и заменяя $u$ на $|t|^{2} / 2$, мы видим, что второе слагаемое в правой части (11) равно

$$
\frac{i^{3}|t|^{3} \alpha_{3}\left(e_{t}\right)}{3 !}\left(1+\gamma \frac{|t|^{2}}{2} e^{|t|^{2} / 2}\right) .
$$

Для любого $u>0$

$$
e^{-u}=1-u+\frac{e^{-\theta u} u^{2}}{2}
$$

где $0<\theta<1$. Умножая это равенство на $e^{u}$ и заменяя $u$ на $|t|^{2} / 2$, мы видим, что первое слагаемое в правой части (11) равно

$$
\left(1-\frac{|t|^{2}}{2}\right) e^{|t|^{2} / 2}=1+\frac{\gamma}{2} \frac{|t|^{4}}{4} e^{|t|^{2} / 2} .
$$

Теперь ясно, что

$$
e^{|t|^{2} / 2} f(t)=1+\frac{i^{3} \alpha_{3}\left(e_{t}\right)|t|^{3}}{3 !}+\gamma e^{|t|^{2} / 2} \frac{\beta_{4}+3}{4 !}|t|^{4}+\gamma e^{|t|^{2} / 2} \frac{\left|\alpha_{3}\right|}{12}|t|^{5},
$$

и первое утверждение леммы доказано.

Воспользовавшись равенством

$$
e^{-u}=1-u+\frac{u^{2}}{2}-\frac{1}{3 !} e^{-\theta u} u^{3}, \quad u>0, \quad 0<\theta<1,
$$

вместо (12), нетрудно убедиться в том, что

$$
\begin{aligned}
e^{|t|^{2} / 2} f(t)= & +\frac{i^{3} \alpha_{3}\left(e_{t}\right)|t|^{3}}{3 !}-\frac{|t|^{4}}{8} e^{|t|^{2} / 2}+\gamma e^{|t|^{2} / 2} \frac{\beta_{4}}{4 !}+\gamma e^{|t|^{2} / 2} \frac{\alpha_{3}\left(e_{t}\right)}{12}|t|^{5} \\
& +\frac{\gamma}{3 !} e^{|t|^{2} / 2}\left(\frac{|t|^{2}}{2}\right)^{3}=1+\frac{i^{3} \alpha_{3}\left(e_{t}\right)|t|^{3}}{3 !}-\frac{|t|^{4}}{8}+\gamma e^{|t|^{2} / 2} \frac{\beta_{4}}{4 !}|t|^{4} \\
& +\gamma e^{|t|^{2} / 2} \frac{\left|\alpha_{3}\right|}{12}|t|^{5}+\gamma e^{|t|^{2} / 2} \frac{|t|^{6}}{12}
\end{aligned}
$$

и второе утверждение леммы доказано. 
Доказательство третьего утверждения леммы проводится аналогично предыдушему, и мы его опускаем.

Д оказатель ст во т еоремы 1. Воспользуемся зарядом (знакопеременной мерой) с характеристической функцией

$$
g(t)=e^{-|t|^{2} / 2} e^{i^{3} \alpha_{3}\left(e_{t}\right)|t|^{3} / 3 !} .
$$

Легко понять, что плотность $q_{n}$ нормированной $n$-кратной свертки этого заряда можно вычислять по формуле обращения

$$
q_{n}(x)=\frac{1}{(2 \pi)^{d}} \int e^{-i(t, x)} g^{n}\left(\frac{t}{\sqrt{n}}\right) d t .
$$

Поэтому при $n \geqslant \nu$

$$
p_{n}(x)-q_{n}(x)=\frac{1}{(2 \pi)^{d}} \int_{|t| \leqslant T \sqrt{n}} e^{-i(t, x)}\left(f^{n}\left(\frac{t}{\sqrt{n}}\right)-g^{n}\left(\frac{t}{\sqrt{n}}\right)\right) d t+R,
$$

где

$$
\begin{aligned}
|R| \leqslant & \frac{1}{(2 \pi)^{d}} \int_{|t| \geqslant T \sqrt{n}}\left|f^{n}\left(\frac{t}{\sqrt{n}}\right)\right| d t+\frac{1}{(2 \pi)^{d}} \int_{|t| \geqslant T \sqrt{n}}\left|g^{n}\left(\frac{t}{\sqrt{n}}\right)\right| d t \\
& +\frac{n^{d / 2}}{(2 \pi)^{d}} \alpha^{n-\nu}(T) \int_{|t| \geqslant T}|f(t)|^{\nu} d t+\frac{1}{(2 \pi)^{d}} \int_{|t| \geqslant T \sqrt{n}} e^{-|t|^{2} / 2} d t
\end{aligned}
$$

а $T>0$ - та же величина, что в (2). Ясно, что при росте $n$ величина $|R|$ убывает экспоненциально быстро.

Первое слагаемое в правой части (13) равно

$$
\begin{gathered}
\sum_{j=0}^{n-1} \frac{1}{(2 \pi)^{d}} \int_{|t| \leqslant T \sqrt{n}} e^{-i(t, x)} f^{n-j-1}\left(\frac{t}{\sqrt{n}}\right) g^{j}\left(\frac{t}{\sqrt{n}}\right)\left(f\left(\frac{t}{\sqrt{n}}\right)-g\left(\frac{t}{\sqrt{n}}\right)\right) d t \\
=\sum_{j=0}^{n-1} \frac{1}{(2 \pi)^{d}} \int_{|t| \leqslant T \sqrt{n}} e^{-i(t, x)} f^{n-j-1}\left(\frac{t}{\sqrt{n}}\right) g^{j}\left(\frac{t}{\sqrt{n}}\right) e^{-|t|^{2} /(2 n)} \\
\times\left(e^{|t|^{2} /(2 n)} f\left(\frac{t}{\sqrt{n}}\right)-e^{|t|^{2} /(2 n)} g\left(\frac{t}{\sqrt{n}}\right)\right) d t
\end{gathered}
$$

В силу первого утверждения леммы при всех $t \in E^{d}$

$$
\begin{aligned}
e^{|t|^{2} /(2 n)} f\left(\frac{t}{\sqrt{n}}\right)= & 1+\frac{i^{3} \alpha_{3}\left(e_{t}\right)|t|^{3}}{3 ! n^{3 / 2}}+\gamma e^{|t|^{2} /(2 n)} \frac{\beta_{4}+3}{4 !} \frac{|t|^{4}}{n^{2}} \\
& +\gamma e^{|t|^{2} /(2 n)} \frac{\left|\alpha_{3}\right|}{12} \frac{|t|^{5}}{n^{5 / 2}}
\end{aligned}
$$

и очевидно, что

$$
e^{|t|^{2} /(2 n)} g\left(\frac{t}{\sqrt{n}}\right)=\exp \left\{\frac{i^{3} \alpha_{3}\left(e_{t}\right)|t|^{3}}{3 ! n^{3 / 2}}\right\}=1+\frac{i^{3} \alpha_{3}\left(e_{t}\right)|t|^{3}}{3 ! n^{3 / 2}}+\frac{\gamma}{2}\left(\frac{\left|\alpha_{3}\right|}{3 !}\right)^{2} \frac{|t|^{6}}{n^{3}} \text {. }
$$


Отсюда и из (2) следует, что правая часть (15) не превосходит

$$
\begin{gathered}
\frac{n}{(2 \pi)^{d}} \int_{|t| \leqslant T \sqrt{n}} \mu^{n-1}\left(\frac{t}{\sqrt{n}}\right)\left(\frac{\beta_{4}+3}{4 !} \frac{|t|^{4}}{n^{2}}+\frac{\left|\alpha_{3}\right|}{12} \frac{|t|^{5}}{n^{5 / 2}}\right) d t \\
\quad+\frac{n}{(2 \pi)^{d}} \int_{|t| \leqslant T \sqrt{n}} \mu^{n}\left(\frac{t}{\sqrt{n}}\right)\left(\frac{1}{2}\left(\frac{\left|\alpha_{3}\right|}{3 !}\right)^{2} \frac{|t|^{6}}{n^{3}}\right) d t \\
=\frac{\beta_{4}+3}{4 !} \frac{B_{4, n-1}}{n}+\frac{\left|\alpha_{3}\right|}{12} \frac{B_{5, n-1}}{n^{3 / 2}}+\frac{1}{2}\left(\frac{\left|\alpha_{3}\right|}{3 !}\right)^{2} \frac{B_{6, n}}{n^{2}} .
\end{gathered}
$$

Сумма правых частей (14) и (16) дает оценку величины $\mid p_{n}(x)-$ $q_{n}(x) \mid$ для всех $x \in E^{d}$.

Очевидно, что функцию $q_{n}(x)$ можно представить в виде

$$
\begin{aligned}
q_{n}(x) & =\frac{1}{(2 \pi)^{d}} \int e^{-i(t, x)} e^{-|t|^{2} / 2} \exp \left\{\frac{i^{3} \alpha_{3}\left(e_{t}\right)|t|^{3}}{3 ! \sqrt{n}}\right\} d t \\
& =\frac{1}{(2 \pi)^{d}} \int e^{-i(t, x)} e^{-|t|^{2} / 2}\left(1+\frac{i^{3} \alpha_{3}\left(e_{t}\right)|t|^{3}}{3 ! \sqrt{n}}\right) d t+R
\end{aligned}
$$

где

$$
|R| \leqslant \frac{1}{(2 \pi)^{d}} \int e^{-|t|^{2} / 2} \frac{1}{2}\left(\frac{\left|\alpha_{3}\right|}{3 !}\right)^{2} \frac{|t|^{6}}{n} d t=\frac{1}{2}\left(\frac{\left|\alpha_{3}\right|}{3 !}\right)^{2} \frac{B_{6}}{n} .
$$

Ясно, что первое слагаемое в правой части (17) равно

$$
\begin{aligned}
& \varphi(x)+\frac{1}{6 \sqrt{n}} \frac{i^{3}}{(2 \pi)^{d}} \int e^{-i(t, x)} e^{-|t|^{2} / 2} \alpha_{3}\left(e_{t}\right)|t|^{3} d t \\
& =\varphi(x)+\frac{1}{6 \sqrt{n}} \frac{i^{3}}{(2 \pi)^{d}} \int e^{-i(t, x)} e^{-|t|^{2} / 2}\left(\int(t, u)^{3} P(d u)\right) d t \\
& =\varphi(x)+\frac{1}{6 \sqrt{n}} \int\left(\frac{i^{3}}{(2 \pi)^{d}} \int e^{-i(t, x)} e^{-|t|^{2} / 2}(t, u)^{3} d t\right) P(d u) \\
& =\varphi(x)+\frac{1}{6 \sqrt{n}} \int H_{3}^{(0)}(x)(u, u, u) \varphi(x) P(d u) .
\end{aligned}
$$

Из полученных соотношений следует утверждение теоремы 1.

Д ок азате льст в о т е о рем ы 2. Первое из утверждений этой теоремы сразу получается, если мы воспользуемся более точным, чем при доказательстве теоремы 1 , асимптотическим разложением плотности $q_{n}(x)$ :

$$
\begin{aligned}
q_{n}(x) & =\frac{1}{(2 \pi)^{d}} \int e^{-i(t, x)} e^{-|t|^{2} / 2} \exp \left\{\frac{i^{3} \alpha_{3}\left(e_{t}\right)|t|^{3}}{3 ! \sqrt{n}}\right\} d t \\
& =\frac{1}{(2 \pi)^{d}} \int e^{-i(t, x)} e^{-|t|^{2} / 2}\left(1+\frac{i^{3} \alpha_{3}\left(e_{t}\right)|t|^{3}}{3 ! \sqrt{n}}+\frac{1}{2} \frac{i^{6} \alpha_{3}^{2}\left(e_{t}\right)|t|^{6}}{(3 ! \sqrt{n})^{2}}\right) d t+R
\end{aligned}
$$

где

$$
|R| \leqslant \frac{1}{(2 \pi)^{d}} \int e^{-|t|^{2} / 2} \frac{1}{3 !}\left(\frac{\left|\alpha_{3}\right|}{3 !}\right)^{3} \frac{|t|^{9}}{n^{3 / 2}} d t=\frac{1}{3 !}\left(\frac{\left|\alpha_{3}\right|}{3 !}\right)^{3} \frac{B_{9}}{n^{3 / 2}} .
$$


Нетрудно понять, что

$$
\begin{aligned}
& \frac{1}{(2 \pi)^{d}} \int e^{-i(t, x)} e^{-|t|^{2} / 2} \frac{1}{2} \frac{i^{6} \alpha_{3}^{2}\left(e_{t}\right)|t|^{6}}{(3 ! \sqrt{n})^{2}} d t \\
& \quad=\frac{1}{72 n} \frac{i^{6}}{(2 \pi)^{d}} \int e^{-i(t, x)} e^{-|t|^{2} / 2}\left(\int(t, u)^{3} P(d u)\right)\left(\int(t, v)^{3} P(d v)\right) d t .
\end{aligned}
$$

Применяя дважды теорему Фубини, мы видим, что правая часть этого равенства есть

$$
\begin{aligned}
& \frac{1}{72 n} \iint\left(\frac{i^{6}}{(2 \pi)^{d}} \int e^{-i(t, x)} e^{-|t|^{2} / 2}(t, u)^{3}(t, v)^{3} d t\right) P(d u) P(d v) \\
& \quad=\frac{1}{72 n} \iint H_{6}^{(0)}(x)(u, u, u, v, v, v) \varphi(x) P(d u) P(d v) .
\end{aligned}
$$

Теперь равенство (5) и оценка (6) очевидны.

Для доказательства соотношений (7) и (8) рассмотрим заряд с характеристической функцией

$$
g(t)=e^{-|t|^{2} / 2} e^{i^{3} \alpha_{3}\left(e_{t}\right)|t|^{3} / 3 !}\left(1-\frac{|t|^{4}}{8}\right) .
$$

Действуя точно так же, как при доказательстве теоремы 1 , нетрудно получить неравенство

$$
\begin{aligned}
\left|p_{n}(x)-q_{n}(x)\right| \leqslant & \frac{n}{(2 \pi)^{d}} \int_{|t| \leqslant T \sqrt{n}} \mu^{n-1}\left(\frac{t}{\sqrt{n}}\right) e^{-|t|^{2} /(2 n)} \\
& \quad \times\left|e^{|t|^{2} /(2 n)} f\left(\frac{t}{\sqrt{n}}\right)-e^{|t|^{2} /(2 n)} g\left(\frac{t}{\sqrt{n}}\right)\right| d t \\
& +\frac{n^{d / 2}}{(2 \pi)^{d}} \alpha^{n-\nu}(T) \int_{|t| \geqslant T}|f(t)|^{\nu} d t \\
& +\frac{1}{(2 \pi)^{d}} \int_{|t| \geqslant T \sqrt{n}}\left|g^{n}\left(\frac{t}{\sqrt{n}}\right)\right| d t
\end{aligned}
$$

где $q_{n}$ - плотность $n$-кратной нормированной свертки заряда с характеристической функцией (18). Очевидно, что последнее слагаемое в правой части (19) не превосходит

$$
\frac{n^{d / 2}}{(2 \pi)^{d}} \beta^{n-1}(T) \int_{|t| \geqslant T} e^{-|t|^{2} / 2}\left|1-\frac{|t|^{4}}{8}\right| d t,
$$

где $\beta(T)=\max \left\{\left.e^{-|t|^{2} / 2}|1-| t\right|^{4} / 8|:| t \mid \geqslant T\right\}<1$, и ясно, что оно при росте $n$ убывает экспоненциально быстро.

В силу второго утверждения леммы и очевидных равенств

$$
\begin{aligned}
e^{|t|^{2} /(2 n)} g\left(\frac{t}{\sqrt{n}}\right) & =\exp \left\{\frac{i^{3} \alpha_{3}\left(e_{t}\right)|t|^{3}}{3 ! n^{3 / 2}}\right\}-\frac{|t|^{4}}{8 n^{2}} \exp \left\{\frac{i^{3} \alpha_{3}\left(e_{t}\right)|t|^{3}}{3 ! n^{3 / 2}}\right\} \\
& =1+\frac{i^{3} \alpha_{3}\left(e_{t}\right)|t|^{3}}{3 ! n^{3 / 2}}+\frac{\gamma}{2}\left(\frac{\left|\alpha_{3}\right|}{3 !}\right)^{2} \frac{|t|^{6}}{n^{3}}-\frac{|t|^{4}}{8 n^{2}}+\frac{|t|^{4}}{8 n^{2}} \gamma \frac{\left|\alpha_{3}\right|}{3 !} \frac{|t|^{3}}{n^{3 / 2}}
\end{aligned}
$$


выражение под знаком модуля в первом слагаемом в правой части (19) не превосходит

$$
e^{|t|^{2} /(2 n)} \frac{\beta_{4}}{4 !} \frac{|t|^{4}}{n^{2}}+e^{|t|^{2} /(2 n)} \frac{\left|\alpha_{3}\right|}{12} \frac{|t|^{5}}{n^{5 / 2}}+e^{|t|^{2} /(2 n)} \frac{|t|^{6}}{12 n^{3}}+\frac{\left|\alpha_{3}\right|^{2}}{72} \frac{|t|^{6}}{n^{3}}+\frac{\left|\alpha_{3}\right|}{48} \frac{|t|^{7}}{n^{7 / 2}} .
$$

Легко понять, что это слагаемое не превосходит

$$
\frac{\beta_{4}}{4 !} \frac{B_{4, n-1}}{n}+\frac{\left|\alpha_{3}\right|}{72} \frac{B_{5, n-1}}{n^{3 / 2}}+\frac{B_{6, n-1}}{12 n^{2}}+\frac{\left|\alpha_{3}\right|^{2}}{72} \frac{B_{6, n}}{n^{2}}+\frac{\left|\alpha_{3}\right|}{48} \frac{B_{7, n}}{n^{5 / 2}} .
$$

Получим теперь асимптотическое разложение плотности

$$
\begin{aligned}
q_{n}(x) & =\frac{1}{(2 \pi)^{d}} \int e^{-i(t, x)} e^{-|t|^{2} / 2} \exp \left\{\frac{i^{3} \alpha_{3}\left(e_{t}\right)|t|^{3}}{3 ! \sqrt{n}}\right\}\left(1-\frac{|t|^{4}}{8 n^{2}}\right)^{n} d t \\
& =\frac{1}{(2 \pi)^{d}} \int e^{-i(t, x)} e^{-|t|^{2} / 2} \exp \left\{\frac{i^{3} \alpha_{3}\left(e_{t}\right)|t|^{3}}{3 ! \sqrt{n}}\right\}\left(1-\frac{|t|^{4}}{8 n}\right) d t+R
\end{aligned}
$$

где

$$
|R| \leqslant \sum_{j=2}^{n} C_{n}^{j} \frac{B_{4 j}}{8^{j} n^{2 j}}
$$

Нетрудно проверить, что при любом $j \geqslant 1$ отношение $B_{4(j+1)} / B_{4 j}$ равно

$$
\int|t|^{4 j+4} e^{-|t|^{2} / 2} d t\left(\int|t|^{4 j} e^{-|t|^{2} / 2} d t\right)^{-1}=(4 j+d+2)(4 j+d)
$$

поэтому в сумме

$$
\sum_{j=2}^{n} C_{n}^{j}\left(\frac{A}{n^{2}}\right)^{j} B_{4 j}=\sum_{j=2}^{n} b_{j}
$$

где $A$ - произвольное положительное число, отношение $b_{j+1} / b_{j}$ равно

$$
\begin{aligned}
& \frac{n-j}{j+1} \frac{A}{n^{2}}(4 j+d+2)(4 j+d) \\
& \quad=\frac{A}{n^{2}} \frac{n-j}{j+1}(16 j(j+1)+8(d-1) j+d(d+2)) \\
& \quad \leqslant A\left(16 \frac{(n-j) j}{n^{2}}+8 \frac{d-1}{n}+\frac{d(d+2)}{3 n}\right)
\end{aligned}
$$

при $j \geqslant 2$. Поскольку максимум функции $(n-x) x, 0 \leqslant x \leqslant n$, равен $n^{2} / 4$, то правая часть неравенства (23) не больше $4 A+8 A(d-1) / n+$ $A d(d+2) /(3 n)=\rho$ и ясно, что если $\rho<1$, то сумму $(22)$ можно оценить суммой геометрической прогрессии со знаменателем $\rho$, поэтому она не превосходит

$$
\frac{1}{1-\rho} C_{n}^{2}\left(\frac{A}{n^{2}}\right)^{2} B_{8} \leqslant \frac{A^{2}}{2(1-\rho)} \frac{B_{8}}{n^{2}} .
$$

Отсюда следует, что правая часть (21) не превосходит

$$
\frac{1}{128(1-\rho)} \frac{B_{8}}{n^{2}}, \quad \text { где } \quad \rho=\frac{1}{2}+\frac{d-1}{n}+\frac{d(d+2)}{24 n},
$$

а эта величина по условию теоремы меньше единицы. 
Рассмотрим, наконец, первое слагаемое в правой части (20). Ясно, что оно равно

$$
\begin{aligned}
& \frac{1}{(2 \pi)^{d}} \int e^{-i(t, x)} e^{-|t|^{2} / 2} \exp \left\{\frac{i^{3} \alpha_{3}\left(e_{t}\right)|t|^{3}}{3 ! \sqrt{n}}\right\} d t \\
& -\frac{1}{(2 \pi)^{d}} \int e^{-i(t, x)} e^{-|t|^{2} / 2} \exp \left\{\frac{i^{3} \alpha_{3}\left(e_{t}\right)|t|^{3}}{3 ! \sqrt{n}} \frac{|t|^{4}}{8 n}\right\} d t .
\end{aligned}
$$

Первое слагаемое, как мы уже видели, равно

$$
\varphi(x)+\frac{\varphi(x)}{6 \sqrt{n}} \int H_{3}^{(0)}(x)(u, u, u) P(d u)+\frac{\varphi(x)}{72 n} H_{6}(x ; P)+R,
$$

где

$$
|R| \leqslant \frac{1}{3 !}\left(\frac{\left|\alpha_{3}\right|}{3 !}\right)^{3} \frac{B_{9}}{n^{3 / 2}} .
$$

Легко понять, что второе слагаемое в (24) равно

$$
-\frac{1}{(2 \pi)^{d}} \int e^{-i(t, x)} e^{-|t|^{2} / 2} \frac{|t|^{4}}{8 n} d t+R=-\frac{\varphi(x)}{8 n} H_{0}^{(4)}(x)+R,
$$

где

$$
|R| \leqslant \frac{\left|\alpha_{3}\right|}{48} \frac{B_{7}}{n^{3 / 2}}
$$

Из полученных соотношений следует утверждение теоремы.

Д ок аз а тель с т в о т е о ремы 3 аналогично доказательству предыдущих утверждений. Рассмотрим заряд с характеристической функцией

$$
g(t)=e^{-|t|^{2} / 2} e^{i^{3} \alpha_{3}\left(e_{t}\right)|t|^{3} / 3 !}\left(1+\frac{\theta_{4}\left(e_{t}\right)|t|^{4}}{4 !}\right)
$$

где $\theta_{4}\left(e_{t}\right)=\alpha_{4}\left(e_{t}\right)-3$. Используя ту же технику, что применялась ранее, а также третье утверждение леммы, нетрудно показать, что

$$
\begin{aligned}
\left|p_{n}(x)-q_{n}(x)\right| \leqslant & \left(\frac{\beta_{5}}{5 !}+\frac{\left|\alpha_{3}\right|}{12}\right) \frac{B_{5, n-1}}{n^{3 / 2}}+\frac{\left|\alpha_{4}\right|+4}{48} \frac{B_{6, n-1}}{n^{2}}+\frac{1}{2}\left(\frac{\left|\alpha_{3}\right|}{3 !}\right)^{2} \frac{B_{6, n}}{n^{2}} \\
& +\frac{\left|\alpha_{3}\right|}{3 !} \frac{\left|\theta_{4}\right|}{4 !} \frac{B_{7, n}}{n^{5 / 2}}+\frac{n^{d / 2}}{(2 \pi)^{d}} \alpha^{n-\nu}(T) \int_{|t| \geqslant T}|f(t)|^{\nu} d t \\
& +\frac{n^{d / 2}}{(2 \pi)^{d}} \beta^{n-1}(T) \int_{|t| \geqslant T} e^{-|t|^{2} / 2}\left(1+\frac{\left|\theta_{4}\right|}{4 !}|t|^{4}\right) d t,
\end{aligned}
$$

где $q_{n}$ - плотность $n$-кратной нормированной свертки заряда с характеристической функцией (25) и

$$
\beta(T)=\max \left\{e^{-|t|^{2} / 2}\left(1+\frac{\left|\theta_{4}\right|}{4 !}|t|^{4}\right):|t| \geqslant T\right\}<1 .
$$

Последнее неравенство заведомо выполнено, если $\beta_{4}<9$. 
Асимптотическое разложение плотности $q_{n}$ получается точно так же, как при доказательстве второй части теоремы 2 , т.е.

$$
\begin{aligned}
q_{n}(x)=\frac{1}{(2 \pi)^{d}} \int & e^{-i(t, x)} e^{-|t|^{2} / 2} \exp \left\{\frac{i^{3} \alpha_{3}\left(e_{t}\right)|t|^{3}}{3 ! \sqrt{n}}\right\} \\
& \times\left(1+\frac{\theta_{4}\left(e_{t}\right)|t|^{4}}{4 ! n}\right) d t+R
\end{aligned}
$$

где

$$
|R| \leqslant \sum_{j=2}^{j}\left(\frac{\left|\theta_{4}\right|}{4 ! n^{2}}\right)^{j} B_{4 j},
$$

и нетрудно понять, что при

$$
\rho=\frac{\left|\theta_{4}\right|}{6}+\frac{\left|\theta_{4}\right|(d-1)}{3 n}+\frac{\left|\theta_{4}\right| d(d+2)}{72 n}<1
$$

правая часть (27) не превосходит

$$
\frac{1}{2(1-\rho)}\left(\frac{\left|\theta_{4}\right|}{4 !}\right)^{2} \frac{B_{8}}{n^{2}} .
$$

Отметим, что при $\beta_{4}<9$ справедливо неравенство $\left|\theta_{4}\right|<6$.

Для завершения доказательства теоремы остается заметить, что первое слагаемое в правой части (26) есть

$$
\begin{aligned}
\varphi(x) & +\frac{\varphi(x)}{6 \sqrt{n}} \int H_{3}^{(0)}(x)(u, u, u) P(d u)+\frac{\varphi(x)}{72 n} H_{6}(x ; P) \\
& +\frac{1}{(2 \pi)^{d}} \int e^{-i(t, x)} e^{-|t|^{2} / 2} \frac{\alpha_{4}\left(e_{t}\right)}{4 !} \frac{|t|^{4}}{n} d t-\frac{\varphi(x)}{8 n} H_{0}^{(4)}(x)+R,
\end{aligned}
$$

где

$$
|R| \leqslant \frac{1}{3 !}\left(\frac{\left|\alpha_{3}\right|}{3 !}\right)^{3} \frac{B_{9}}{n^{3 / 2}}+\frac{\left|\alpha_{3}\right|}{3 !} \frac{\left|\theta_{4}\right|}{4 !} \frac{B_{7}}{n^{3 / 2}}
$$

а второй интеграл в (28) равен

$$
\begin{aligned}
& \frac{1}{(2 \pi)^{d}} \int e^{-i(t, x)} e^{-|t|^{2} / 2}\left(\frac{1}{4 ! n} \int(t, u)^{4} P(d u)\right) d t \\
& =\frac{1}{4 ! n} \int\left(\frac{1}{(2 \pi)^{d}} \int e^{-i(t, x)} e^{-|t|^{2} / 2}(t, u)^{4} d t\right) P(d u) \\
& =\frac{1}{4 ! n} \int H_{4}(x)(u, u, u, u) \varphi(x) P(d u) .
\end{aligned}
$$

Действуя аналогично тому, как мы действовали при переходе от теоремы 1 к теореме 2, можно получить разложения, остаточные члены которых оцениваются лучше, чем в теореме 3 , во всяком случае при больших $n$. При этом в главных частях разложений появятся величины, связанные с многочленами Чебышёва-Эрмита девятого и седьмого порядков. В случае, когда конечна величина $\beta_{6}$, можно получить 
разложение, главная часть которого содержит величины, связанные с многочленами Чебышёва-Эрмита пятого, седьмого и девятого порядков. Эти величины при росте $n$ убывают как $1 / n^{3 / 2}$, а остаточная часть как $1 / n^{2}$. Эти разложения оказываются очень громоздкими, что связано, главным образом, с тем, что громоздкими являются величины, связанные с многочленом Чебышёва-Эрмита девятого порядка. Быть может, стоит отметить, что многочлен $H_{9}^{(0)}(x)\left(h_{1}, \ldots, h_{9}\right)$ является суммой $2620(!)$ различных слагаемых, многочлен $H_{6}^{(0)}(x)\left(h_{1}, \ldots, h_{6}\right)$ есть сумма 76 , а $H_{4}^{(0)}(x)\left(h_{1}, h_{2}, h_{3}, h_{4}\right)$ - сумма 10 слагаемых. Явный вид многочлена $H_{4}^{(0)}(x)\left(h_{1}, h_{2}, h_{3}, h_{4}\right)$ приведен в [1]. Количество слагаемых в многочлене $H_{l}^{(0)}(x)\left(h_{1}, \ldots, h_{l}\right)$ совпадает с суммой абсолютных величин коэффициентов в одномерном многочлене Чебышёва-Эрмита $l$-го порядка.

\section{СПИСОК ЛИТЕРАТУРЫ}

1. Сенатов В. В. Об одном многомерном аналоге разложения Чебышёва. - Теория вероятн. и ее примен., 2006, т. 52 , в. 3, с. 603-610.

2. Сенатов $B$. . О. Об асимптотических разложениях в центральной предельной теореме с явными оценками остаточных членов. - Теория вероятн. и ее примен., 2005 , т. 51 , в. 4 , с. $810-816$. 